\title{
Strong and weak chaos in networks of semiconductor lasers with time-delayed couplings
}

\author{
Sven Heiligenthal, ${ }^{1}$ Thomas Jüngling, ${ }^{1,2}$ Otti D’Huys, ${ }^{1}$ Diana A. Arroyo-Almanza, ${ }^{2, *}$ Miguel C. Soriano, ${ }^{2}$ Ingo Fischer, ${ }^{2}$ \\ Ido Kanter, ${ }^{3}$ and Wolfgang Kinzel ${ }^{1}$ \\ ${ }^{1}$ Institute of Theoretical Physics, University of Würzburg, 97074 Würzburg, Germany \\ ${ }^{2}$ Instituto de Física Interdisciplinar y Sistemas Complejos, IFISC (UIB-CSIC), \\ Campus Universitat de les Illes Balears, 07122 Palma de Mallorca, Spain \\ ${ }^{3}$ Department of Physics, Bar-Ilan University, 52900 Ramat-Gan, Israel
}

(Received 5 October 2012; revised manuscript received 15 April 2013; published 8 July 2013)

\begin{abstract}
Nonlinear networks with time-delayed couplings may show strong and weak chaos, depending on the scaling of their Lyapunov exponent with the delay time. We study strong and weak chaos for semiconductor lasers, either with time-delayed self-feedback or for small networks. We examine the dependence on the pump current and consider the question of whether strong and weak chaos can be identified from the shape of the intensity trace, the autocorrelations, and the external cavity modes. The concept of the sub-Lyapunov exponent $\lambda_{0}$ is generalized to the case of two time-scale-separated delays in the system. We give experimental evidence of strong and weak chaos in a network of lasers, which supports the sequence of weak to strong to weak chaos upon monotonically increasing the coupling strength. Finally, we discuss strong and weak chaos for networks with several distinct sub-Lyapunov exponents and comment on the dependence of the sub-Lyapunov exponent on the number of a laser's inputs in a network.
\end{abstract}

DOI: 10.1103/PhysRevE.88.012902

PACS number(s): 05.45.Xt, 89.75.-k, 02.30.Ks

\section{INTRODUCTION}

In the interdisciplinary science of complex systems one often encounters large dynamical systems described as networks of nonlinear units. Examples range from neural over social networks to technological applications with networks of coupled lasers [1-5]. The dynamical units of the network exchange information with a finite propagation velocity, which results in time delays in the transmission. Especially in the case of coupled semiconductor lasers, the delay time is typically much larger than the characteristic time scales of the intensity dynamics. Hence the study of delay-coupled dynamical systems is currently an active field of research $[6,7]$.

Time-delayed feedback on a single system may already generate instabilities leading to deterministic chaos [8-10]. As an example, the emission of a semiconductor laser can become unstable and develop chaotic fluctuations of the light intensity if the laser beam is reflected back into the device by a distant mirror. Networks of nonlinear units may similarly become chaotic due to time-delayed coupling of the nodes [11].

If all units are identical, which means in practice that they are sufficiently similar, the network can synchronize onto a common trajectory. Even if the delay time is extremely large, complete (zero-lag) synchronization is possible [12-14]. Chaos synchronization has been discussed in the context of secure communication $[15,16]$.

Recently, two different kinds of chaos have been identified for chaotic networks of time-continuous systems with timedelayed couplings: strong and weak chaos [17]. In the limit of large delay times, the maximal Lyapunov exponent (LE) of the network saturates at a nonzero value for strong chaos, whereas it scales with the inverse delay time for weak chaos. A similar phenomenon has been reported for time-discrete

\footnotetext{
*Also at: Centro de Investigaciones en Óptica, León 37150, Guanajuato, Mexico.
}

maps with delay [9]. Also for steady states and periodic orbits the Floquet exponents show similar scaling properties [18,19]. Only networks exhibiting weak chaos can synchronize to a common chaotic trajectory.

In this paper we extend the investigations on strong and weak chaos presented in Ref. [17] by focusing on the dynamics of semiconductor lasers. In Sec. II we complete the discussion of the scaling behavior of the LE for a single chaotic unit with delayed self-feedback in the limit of large delays, which has been sketched in Ref. [17]. This system is investigated in Sec. III for semiconductor lasers. As presented in Ref. [17], numerical simulations of the Lang-Kobayashi equations yield the transition from weak to strong chaos and back to weak chaos upon monotonically increasing the coupling strength. We extend this result by discussing the scaling just at the transition and the dependence of the scaling on the laser pump current. Autocorrelations, spatial representations of the chaotic intensity, and external cavity modes are calculated for our additional investigation and we follow the question of whether one can deduce the type of chaos from a single trajectory. An extension of the criterion for the occurrence of strong and weak chaos is given for the case of two time-scale-separated selffeedbacks. In Sec. IV networks of semiconductor lasers are considered. The relation of the stability of the synchronization manifold to the eigenvalue gap of the coupling matrix and the master stability function is specialized for semiconductor lasers. We report on an experiment on semiconductor lasers that supports the sequence of weak to strong to weak chaos with increasing coupling strength. Finally, we present a generalized investigation of networks with several distinct sub-LEs and certain network patterns.

\section{SCALING OF THE MAXIMAL LYAPUNOV EXPONENT}

We first consider a single chaotic unit with time-delayed self-feedback and derive general properties of the LE in the 
limit of large delay times, extending the scaling argument of our earlier work [17]. Our general model reads

$$
\dot{\mathbf{x}}=\mathbf{F}(\mathbf{x})+\sigma \mathbf{H}\left(\mathbf{x}_{\tau}\right),
$$

where $\mathbf{x} \equiv \mathbf{x}(t) \in \mathbb{R}^{M}$ is the state vector with $M$ degrees of freedom and $\mathbf{x}_{\tau} \equiv \mathbf{x}(t-\tau)$ with the time delay $\tau>0$. The field $\mathbf{F}$ describes the nondelayed part of the nonlinear dynamical system, $\mathbf{H}$ is a coupling function, and $\sigma$ is the overall coupling strength. The linearization of the delay system Eq. (1) is given by

$$
\dot{\delta} \mathbf{x}=D F(\mathbf{x}) \boldsymbol{\delta} \mathbf{x}+\sigma D H\left(\mathbf{x}_{\tau}\right) \boldsymbol{\delta} \mathbf{x}_{\tau}
$$

and can be used to calculate LEs for a given trajectory $\mathbf{x}(t)$, which is obtained by integration of Eq. (1). The maximal LE $\lambda_{m}$ is defined by

$$
\lambda_{m}=\lim _{t \rightarrow \infty} \frac{1}{t} \ln \left\{\frac{\|\delta \mathbf{x}(t)\|}{\left\|\mathbf{\delta} \mathbf{x}\left(t_{0}\right)\right\|}\right\} .
$$

Since the system under investigation is a delay system, we formally obtain infinitely many LEs. In practice, after some transient time, the system relaxes onto the most unstable mode revealing the maximal exponent.

One can also define a conditional LE for delay systems, in analogy to drive-response systems. In a drive-response setup (where the drive is denoted by $\mathcal{D}$ and the response by $\mathcal{R}$ ), this conditional LE $\lambda_{\mathcal{R}}$ describes the evolution of a perturbation applied to the response system $\mathcal{R}$ [20]. It relates to the synchronization properties of the system: If the conditional LE is negative, $\mathcal{R}$ is in a state of generalized synchronization with $\mathcal{D}$. Since the conditional LE relates to a subsystem, it is often called a sub-Lyapunov exponent. The Abarbanel setup [21] for testing the sign of $\lambda_{0}$ is shown in Fig. 1. Considering a delay system as a nondelayed dynamical unit $\mathcal{A} \widehat{=} \mathcal{R}$ driven by a transmission line $\mathcal{A}_{\tau} \widehat{=} \mathcal{D}$, the sub-Lyapunov exponent of the unit arises from the instantaneous part of the equations of motion and therefore has been referred to as an instantaneous Lyapunov exponent [17]. However, in this paper we keep the notion of sub-Lyapunov exponent and denote it as $\lambda_{0}$.

We obtain $\lambda_{0}$ by integrating the linearization

$$
\begin{aligned}
\dot{\delta} \mathbf{x}_{\mathbf{0}} & =D F(\mathbf{x}) \boldsymbol{\delta} \mathbf{x}_{\mathbf{0}}, \\
\lambda_{0} & =\lim _{t \rightarrow \infty} \frac{1}{t} \ln \left\{\frac{\left\|\boldsymbol{\delta} \mathbf{x}_{\mathbf{0}}(t)\right\|}{\left\|\boldsymbol{\delta} \mathbf{x}_{\mathbf{0}}\left(t_{0}\right)\right\|}\right\} .
\end{aligned}
$$

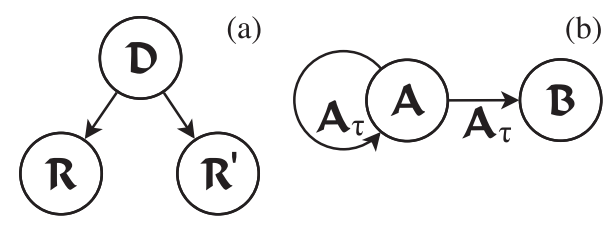

FIG. 1. Test setups for conditional Lyapunov exponents. (a) Abarbanel test for generalized synchronization between $\mathcal{D}$ and $\mathcal{R}$ with test unit $\mathcal{R}^{\prime}$. If $\mathcal{R}$ and $\mathcal{R}^{\prime}$ synchronize completely, $\mathcal{D}$ and $\mathcal{R}$ are in a state of generalized synchronization. (b) Test for strong and weak chaos corresponding to the Abarbanel test such that $\mathcal{A}_{\tau} \widehat{=} \mathcal{D}$, $\mathcal{A} \widehat{=} \mathcal{R}$, and $\mathcal{B} \widehat{=} \mathcal{R}^{\prime}$. If $\mathcal{A}$ and $\mathcal{B}$ synchronize completely, $\mathcal{A}$ is in a state of weak chaos.
Alternatively, we can define $\lambda_{0}$ via the evolution operator of Eq. (4),

$$
\boldsymbol{\delta} \mathbf{x}_{\mathbf{0}}(t)=U\left(t, t_{0}\right) \boldsymbol{\delta} \mathbf{x}_{\mathbf{0}}\left(t_{0}\right),
$$

with $U\left(t_{0}, t_{0}\right)=\mathbb{1}$. Then

$$
\lambda_{0}=\max _{i} \lim _{t \rightarrow \infty} \frac{1}{2 t} \ln \left[\operatorname{eig}_{i}\left\{U^{\top}\left(t, t_{0}\right) U\left(t, t_{0}\right)\right\}\right] .
$$

It is important to mention that although the delay term is skipped in the defining equation for $\lambda_{0}$, the feedback parameters $\sigma$ and $\tau$ enter indirectly via the trajectory $\mathbf{x}(t)$, which is inserted in the linearization (4). It is possible to measure the sign of the sub-LE $\lambda_{0}$ using an auxiliary system approach [17], in analogy to the Abarbanel test for general synchronization [21].

If $\lambda_{0}>0$, we call the resulting chaotic dynamics strong chaos; otherwise, if $\lambda_{0}<0$ but the delay system is still chaotic, we define weak chaos. As we have documented in recent work [17], the sign of $\lambda_{0}$ has two major implications. First, for a single-delay system it determines the scaling of the maximal LE with the delay time. Second, for a network of delay-coupled units it determines the possibility to synchronize the units. If the units are strongly chaotic, the delayed coupling cannot compensate for the exponential divergence of two nearby trajectories of any two systems in the network. In contrast, for weak chaos, synchronization is in principle possible and depends on the network topology.

(a) Strong chaos $\lambda_{0}>0$. In order to derive a scaling relation $\lambda_{m}(\tau)$, we have to assume that $\lambda_{0}(\tau)=$ const. In a strict sense this is never true, but for sufficiently large $\tau$ one always observes a saturation effect and a decrease of remaining fluctuations in $\lambda_{0}(\tau)$, so the assumption of a constant value is valid. We start from the linearization (2). If $\lambda_{0}>0$, for large $\tau$ the instantaneous term becomes dominant and the delay term becomes negligible. This can be seen from the coordinate transformation

$$
\delta \mathbf{x}(t)=e^{\lambda_{0} t} \boldsymbol{\delta} \mathbf{z}(t)
$$

which results in

$$
\dot{\delta} \mathbf{z}=\left[D F(\mathbf{x})-\lambda_{0} \cdot \mathbb{1}\right] \delta \mathbf{z}+\sigma e^{-\lambda_{0} \tau} D H\left(\mathbf{x}_{\tau}\right) \delta \mathbf{z}_{\tau} .
$$

The resulting Lyapunov exponent of the transformed system can therefore be estimated as $\lambda_{\mathbf{z}} \approx 0$. This implies that in the original coordinates $\lambda_{m} \approx \lambda_{0}$, meaning that the LE becomes independent of $\tau$ for large delays.

(b) Weak chaos $\lambda_{0}<0$. In this case we can estimate the scaling of the LE by considering a stroboscopic sequence of the evolution of our linear system $\delta \mathbf{x}_{\mathbf{n}}(\theta)=\delta \mathbf{x}(\theta+n \tau)$ with $n \in \mathbb{N}$ and $\theta \in]-\tau, 0]$. One can introduce a growth factor (Lyapunov multiplier) by $\left\|\boldsymbol{\delta} \mathbf{x}_{\mathbf{n}+\mathbf{1}}\right\|=\mu_{n}\left\|\boldsymbol{\delta} \mathbf{x}_{\mathbf{n}}\right\|$, so that the LE becomes

$$
\lambda_{m}=\lim _{l \rightarrow \infty} \frac{1}{l \tau} \sum_{n=1}^{l} \ln \mu_{n}=\frac{1}{\tau} \ln C,
$$

where $C$ is the geometric mean of all multipliers. In the following, we show that for sufficiently large delay times the multipliers $\mu_{n}$ do not depend on $\tau$, hence the LE is of order $\tau^{-1}$. This means that in weak chaos a perturbation of the chaotic system grows on the time scale of the delay time. Thus we 
introduce the variation-of-constants formula, which provides an integral version of our initial delay differential equation (2), and evaluate it at $\theta=0$,

$$
\begin{aligned}
\delta \mathbf{x}_{\mathbf{n}+\mathbf{1}}(0)= & U_{n}(0,-\tau) \boldsymbol{\delta} \mathbf{x}_{\mathbf{n}}(0) \\
& +\sigma \int_{-\tau}^{0} d t U_{n}(0, t) D H\left[\mathbf{x}_{\mathbf{n}}(t)\right] \boldsymbol{\delta} \mathbf{x}_{\mathbf{n}}(t) .
\end{aligned}
$$

It contains the evolution operator $U_{n}\left(t_{2}, t_{1}\right)$ of the auxiliary system (4) on the $n$th $\tau$ interval. This operator is exponential in $t_{2}-t_{1}$, i.e., with respect to a suitable matrix norm it holds that for $t_{2}>t_{1}$

$$
\left\|U\left(t_{2}, t_{1}\right)\right\|<U_{0} \exp \left[\alpha\left(t_{2}-t_{1}\right)\right],
$$

with $\lambda_{0}<\alpha<0$. The bound provided by $U_{0}$ and $\alpha$ should cover potential bursts typical of the linearization of a chaotic flow. Because of this exponential bound, there is some time $\tau_{0} \propto \alpha^{-1}$, such that the term with $U(0,-\tau)$ can be neglected in Eq. (11), if $\tau>\tau_{0}$. Additionally, the integral has only significant contributions in a small range close to the end of the integration interval, namely, for $t \in\left[-\tau_{0}, 0\right]$. This is due to the fact that none of the other factors in the integrand evolves exponentially in such a way that the exponential decay as depicted in Eq. (12) could be compensated for. The factor $D H\left[\mathbf{x}_{\mathbf{n}}(t)\right]$ is constant on average and the term $\delta \mathbf{x}_{\mathbf{n}}(t)$ does not counteract the evolution operator due to the positive LE considered here. This means that a further increase of $\tau$ beyond $\tau_{0}$ does not affect the integral and the multiplier introduced above can be estimated by

$$
\mu_{n}=\frac{\sigma}{\left\|\delta \mathbf{x}_{\mathbf{n}}(0)\right\|}\left\|\int_{-\tau_{0}}^{0} d t U_{n}(0, t) D H\left[\mathbf{x}_{\mathbf{n}}(t)\right] \delta \mathbf{x}_{\mathbf{n}}(t)\right\|+O\left(e^{-\alpha \tau}\right) .
$$

In leading order, this expression does not depend on the delay time. It depends on $\lambda_{0}, \sigma$, and a set $\mathbf{q}$ of other (yet unknown) statistical properties of the chaotic trajectory. Hence we can write

$$
\lambda_{m}=\frac{1}{\tau} \ln C\left(\lambda_{0}, \sigma, \mathbf{q}\right)
$$

This can be compared to a Floquet problem, in which the driving trajectory is $\tau$ periodic $[\mathbf{x}(t)=\mathbf{x}(t+\tau)]$ and the coupling is linear and diagonal $(D H[\mathbf{x}(t)] \equiv \mathbb{1})$. One obtains $C=-\sigma / \lambda_{0}$.

\section{SINGLE LASER WITH TIME-DELAYED SELF-FEEDBACK}

We demonstrate the occurrence of strong and weak chaos in a paradigmatic system with large delay time: a semiconductor laser with delayed self-feedback. Here we briefly revisit and extend some results discussed in our earlier work [17]. Our system is modeled by the Lang-Kobayashi (LK) equations

$$
\begin{aligned}
& \dot{\mathcal{E}}(t)=\frac{1+i \alpha}{2} G_{N} n(t) \mathcal{E}(t)+\sigma \exp \left(-i \omega_{0} \tau\right) \mathcal{E}(t-\tau), \\
& \dot{n}(t)=(p-1) J_{\mathrm{th}}-\gamma n(t)-\left[\Gamma+G_{N} n(t)\right]|\mathcal{E}(t)|^{2},
\end{aligned}
$$

where $\mathcal{E}(t)$ and $n(t)$ denote the complex electric field and the excess carrier density, respectively. The feedback is characterized by a delay $\tau$ and a strength $\sigma$. The parameters
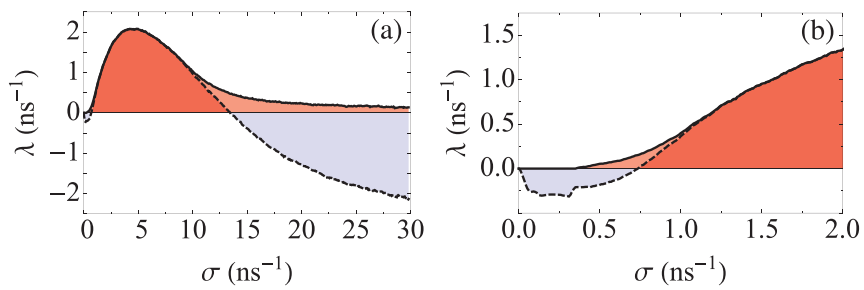

FIG. 2. (Color online) (a) Maximal LE $\lambda_{m}$ (solid line) and sub-LE $\lambda_{0}$ (dashed line) of a single laser with self-feedback for a delay time $\tau=10 \mathrm{~ns}$ vs coupling strength $\sigma$. (b) Enlarged view of (a) for small coupling strengths $\sigma$. Both figures have been adapted from Ref. [17].

involved can be found in Table I in Appendix A. We have omitted the intensity-dependent nonlinear gain saturation. Nevertheless, for the applied pump currents all results coincide qualitatively with the results of the complete rate equations. Unless stated otherwise, all diagrams are made for a delay time of $\tau=10 \mathrm{~ns}$ and a pump current of $p=1.02$.

We calculate the maximal LE $\lambda_{m}$ and the sub-LE $\lambda_{0}$ according to Eqs. (1)-(5). In order to approximate the formal limits as well as possible with a finite simulation run, we typically choose an integration period of 1000 delay times. If huge fluctuations occur on the linearization during this interval, we also average over an ensemble of trajectories with different initial conditions. The chosen interval length also covers sufficiently many cycles of the typical low-frequency fluctuations occurring in feedback lasers. Instead of using the usual norms for $\|\boldsymbol{\delta} \mathbf{x}(t)\|$ (see, e.g., Ref. [8]), we restricted the evaluation on the electric field component and considered only $|\delta \mathcal{E}(t)|$, which is no longer a norm in the formal sense, but still yields very low fluctuations of the final exponent. The nonvanishing matrix elements in $D F[\mathbf{x}(t)]$ connecting $\delta \mathcal{E}$ and $\delta n$ guarantee in all regimes of the LK equations studied here that the leading Lyapunov vector affects the $\delta \mathcal{E}$ components. Thus the obtained LE is the same as if also $\delta n$ were present in the measure of $\delta \mathbf{x}(t)$. Numerical calculations with different (semi)norms confirm this property for examples in both strong and weak chaos.

Figure 2 shows $\lambda_{m}$ (solid line) and $\lambda_{0}$ (dashed line) of a single laser with self-feedback dependent on the coupling strength $\sigma$. We observe a transition from periodic behavior (Goldstone mode with $\lambda_{m}=0$ ) to weak chaos and from there transitions to strong chaos and back to weak chaos upon monotonically increasing the coupling strength.

Figure 3 shows the different behaviors of the maximal LE and the sub-LE for the regimes of weak [Fig. 3(a)] and strong chaos [Fig. 3(b)] dependent on the delay time $\tau$. As soon as the delay time is large compared with the internal time scales of the laser, the sub-LE $\lambda_{0}$ remains constant with increasing $\tau$ for both weak and strong chaos. For weak chaos, $\lambda_{m}$ decreases like $1 / \tau$ for growing $\tau$. For strong chaos, $\lambda_{m}$ converges exponentially to the positive sub-LE $\lambda_{0}$.

The product $\lambda_{m} \tau$ is the relevant dimensionless quantity describing chaoticity in systems with delay. For weak chaos this product as a function of $\tau$ has two properties. First, it saturates at a constant value $C$ that depends on the coupling strength [Fig. 3(c)]. According to the analytical calculations underlying Eq. (14), this dependence is primarily caused by different values of the sub-LE $\lambda_{0}(\sigma)$ as shown in Fig. 3(a). 

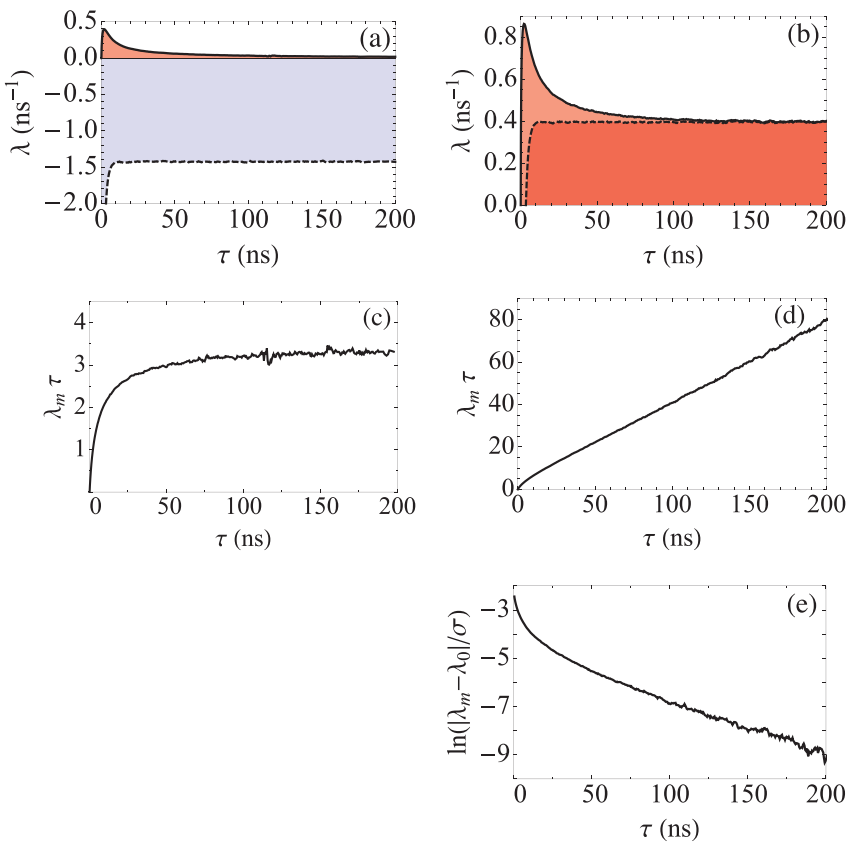

FIG. 3. (Color online) Maximal LE $\lambda_{m}$ (solid line) and sub-LE $\lambda_{0}$ (dashed line) of a single laser with self-feedback for (a) weak chaos ( $\left.\sigma=21 \mathrm{~ns}^{-1}\right)$ and (b) strong chaos $\left(\sigma=12 \mathrm{~ns}^{-1}\right)$ vs delay time $\tau$. (c) Product $\lambda_{m} \tau$ of a single laser with self-feedback vs delay time $\tau$ for weak chaos $\left(\sigma=21 \mathrm{~ns}^{-1}\right)$ with onset of saturation at $\tau_{\text {sat }} \approx 50 \mathrm{~ns}$. (d) $\lambda_{m} \tau$ for strong chaos $\left(\sigma=12 \mathrm{~ns}^{-1}\right)$. (e) $\ln \left(\left|\lambda_{m}-\lambda_{0}\right| / \sigma\right)$ of a single laser with self-feedback vs delay time $\tau$ for strong chaos $\left(\sigma=12 \mathrm{~ns}^{-1}\right)$. (c) and (e) have been adapted from Ref. [17].

Second, the delay time $\tau_{\text {sat }}$ for which the product $\lambda_{m} \tau_{\text {sat }}$ reaches the saturation value up to a fixed distance $\delta$ depends on $\sigma$ : The closer to the critical coupling strength $\sigma_{\text {crit }}$ where $\lambda_{0}=0$, the larger the saturation delay $\tau_{\text {sat }}$.

For strong chaos, on the contrary, $\lambda_{m} \tau$ grows linearly with increasing $\tau$ [Fig. 3(d)] since $\lambda_{m}$ becomes constant, as shown in Fig. 3(b). Figure 3(e) confirms that the convergence $\lambda_{m} \rightarrow$ $\lambda_{0}>0$ happens exponentially with $\tau$. The characteristic exponent of this convergence, however, is much larger (i.e., the convergence is slower) than one would expect from analytic calculations for a simple model with constant coefficients. This effect is caused by the fluctuations of $D F[\mathbf{x}(t)]$, which act like multiplicative noise in Eqs. (2) and (4) [22].
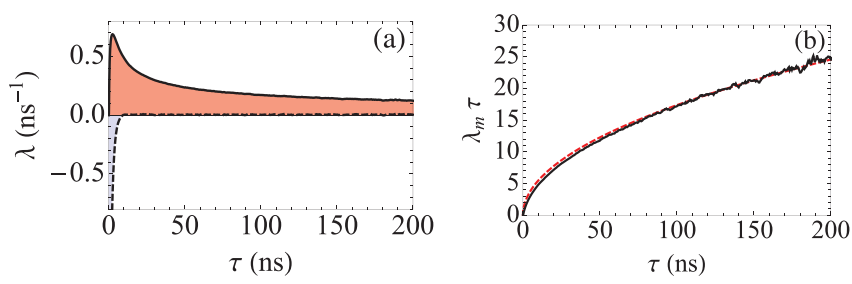

FIG. 4. (Color online) (a) Maximal LE $\lambda_{m}$ (solid line) and sub-LE $\lambda_{0}$ (dashed line) and (b) product $\lambda_{m} \tau$ (black solid line) of a single laser with self-feedback in comparison with the least-squares fit of $\sqrt{\tau}$ (red dashed line) for critical coupling strength where the transition between strong and weak chaos occurs $\left(\sigma_{\text {crit }}=13.4 \mathrm{~ns}^{-1}\right)$ vs delay time $\tau$.

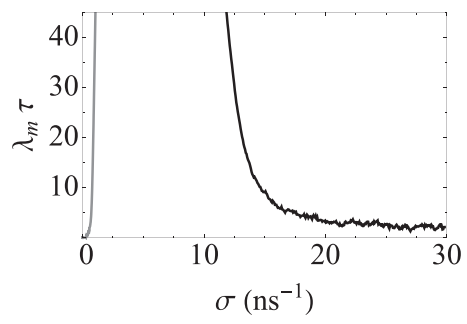

FIG. 5. Product $\lambda_{m} \tau$ of a single laser with self-feedback vs coupling strength $\sigma$ for a delay time $\tau=100 \mathrm{~ns}$. Figure has been adapted from Ref. [17].

Figure 4 shows the special limit case between strong and weak chaos when the sub-LE $\lambda_{0}=0$ for sufficiently large delay times $\tau$. In Fig. 4(a) one can see that the maximal LE still decays with increasing $\tau$. However, it does so very slowly. Figure 4(b) shows the consequence for the product $\lambda_{m} \tau$ at the critical point: It neither grows linearly with $\tau$ like for strong chaos nor does it saturate at a constant value for finite delay times like for weak chaos. From the viewpoint of the saturation behavior for weak chaos, the delay time $\tau_{\text {sat }}$, at which the product $\lambda_{m} \tau$ saturates, has been shifted towards infinity at the critical point. The product $\lambda_{m} \tau$ rather tends to grow like $\sqrt{\tau}$, as shown by the least-squares fit with $\sqrt{\tau}$ (red dashed line) in Fig. 4(b). A rigorous explanation of this scaling behavior is still missing, but in comparison with a simplified linear model, in which the fluctuations of $D F[\mathbf{x}(t)]$ are replaced by multiplicative noise, the emergence of the $\sqrt{\tau}$ scaling is a natural consequence of a restricted random walk [22].

Figure 5 shows that for weak chaos and a very large delay time $\tau \rightarrow \infty$, the product $\lambda_{m} \tau$ undergoes a phase transition and diverges in the proximity of the critical coupling strengths $\sigma_{\text {crit, } 1}$ (gray line) and $\sigma_{\text {crit,2 }}$ (black line). We were able to find transitions between strong and weak chaos by changing the coupling strength $\sigma$ also for the Rössler and Lorenz dynamics. The Stuart-Landau, FitzHugh-Nagumo, and continuous Ikeda dynamics show only weak chaos.

\section{A. Scaling of the sub-Lyapunov exponent with the laser pump current}

We study the dependence of the sub-LE on the laser pump current. Figure 6(a) shows the sub-LE as a function of the feedback strength $\sigma$ for different values of the pump current. We find that the curves all follow the same pattern described in the preceding section. The sub-LE is negative for small and large values of the feedback strength. The agreement is even quantitative. We find that the sub-LE and coupling strength scale with the square root of the effective pump current $\sqrt{p-1}$ above the lasing threshold. Figure 6(b) shows the maximum of the sub-LE $\lambda_{0, \max }$, the feedback strength $\sigma_{0, \max }$ for which the sub-LE is maximal, and the critical feedback strength $\sigma_{0 \text {,crit }}$, where $\lambda_{0}=0$ as a function of the effective pump current $p-1$. The data are shown on a double-logarithmic scale, indicating a slope of 1/2. In Fig. 6(c) the sub-LEs are rescaled with $\sqrt{p-1}$. We find that this scaling relation holds well for small coupling strengths. For larger coupling strengths the sub-LEs diverge for different pump currents. 

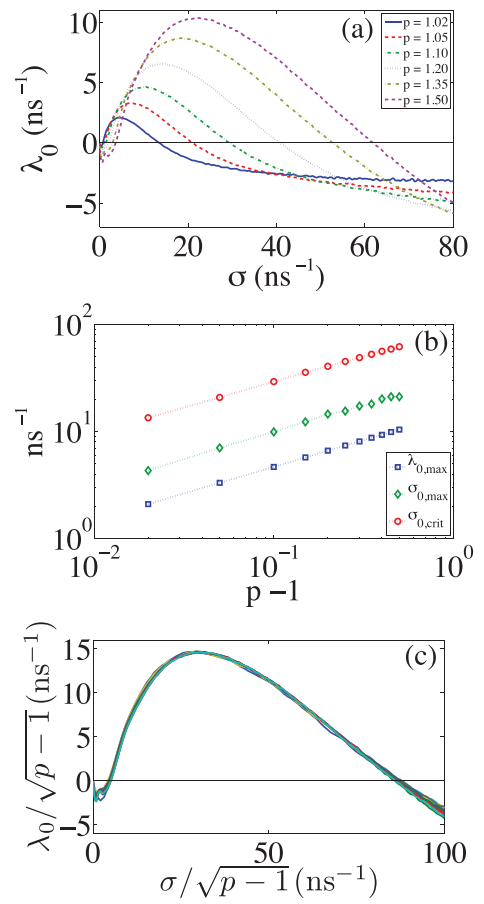

FIG. 6. (Color online) (a) Sub-LE $\lambda_{0}$ of a single laser with self-feedback for a delay time $\tau=10 \mathrm{~ns}$ and several different pump currents $p$ vs coupling strength $\sigma$. (b) Maxima $\lambda_{0, \max }$ of the sub-LEs $\lambda_{0}$, coupling strengths $\sigma_{0, \max }$ of these maxima, and critical coupling strengths $\sigma_{\text {crit }}$, where $\lambda_{0}=0$ for several different pump currents $p$ vs effective pump current $p-1$ on a double-logarithmic scale. (c) Data collapse of rescaled sub-LEs $\lambda_{0} / \sqrt{p-1}$ vs rescaled coupling strengths $\sigma / \sqrt{p-1}$ for 11 different pump currents $p$ ranging from $p=1.02$ to 1.50 .

To explain this scaling behavior, we introduce the following rescaling of the laser parameters:

$$
\begin{aligned}
N & =\sqrt{\frac{G_{N}}{\Gamma N_{\mathrm{sol}}}} \frac{n}{\sqrt{p-1}}, \\
E & =\sqrt{\frac{\Gamma}{\gamma N_{\mathrm{sol}}} \frac{\mathcal{E}}{\sqrt{p-1}},} \\
K & =\frac{1}{\sqrt{G_{N} N_{\mathrm{sol}} \Gamma}} \frac{\sigma}{\sqrt{p-1}}, \\
s & =\sqrt{\Gamma G_{N} N_{\mathrm{sol}}} \sqrt{p-1} t,
\end{aligned}
$$

with $J_{\text {th }}=\gamma N_{\text {sol }}$. Such a scaling reproduces the scaling behavior that we found numerically: The coupling strength $K$ and the Lyapunov exponent (which scales inversely with time) scale with $\sqrt{p-1}$. The LK equations (15) can then be rewritten as

$$
\begin{aligned}
& \frac{d E}{d s}=\frac{1+i \alpha}{2} N E+K e^{i \theta} E(s-\tilde{\tau}), \\
& \frac{d N}{d s}=\frac{\gamma}{\Gamma}\left[1-\frac{N}{c \sqrt{p-1}}-(c \sqrt{p-1} N+1)|E|^{2}\right],
\end{aligned}
$$

with $c=\sqrt{G_{N} N_{\text {sol }} / \Gamma}$ and $\tilde{\tau}=\sqrt{\Gamma G_{N} N_{\text {sol }}} \sqrt{p-1} \tau$. We assume small coupling strength $K \ll 1$, small carrier densities $N=O(K)$, and a reasonably high pump current $c \sqrt{p-1}=$ $O(1)$. The ratio of photon and carrier lifetimes $\gamma / \Gamma$ is a small
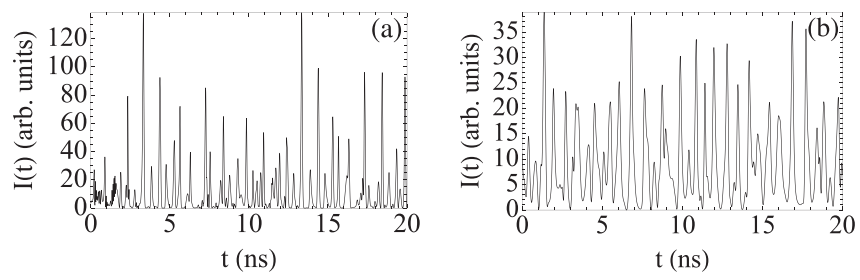

FIG. 7. Example trajectories (intensity traces) of a single laser with a self-feedback of $\tau=10 \mathrm{~ns}$ for (a) weak chaos $\left(\sigma=21 \mathrm{~ns}^{-1}\right)$ and (b) strong chaos $\left(\sigma=6 \mathrm{~ns}^{-1}\right)$ vs time $t$.

parameter as well. In leading order, we obtain the following equations:

$$
\begin{aligned}
& \frac{d E}{d s}=\frac{1+i \alpha}{2} N E+K e^{i \theta} E(s-\tilde{\tau}), \\
& \frac{d N}{d s}=\frac{\gamma}{\Gamma}\left(1-|E|^{2}\right) .
\end{aligned}
$$

These equations only depend on the pump current through the value of the time delay. Since for large delays the exact value of the time delay does not influence the sub-LE, we recover the scaling behavior found numerically. For increasing coupling strength $K$, the mapping becomes less exact, as can be seen in Fig. 6(c). This results from the fact that the rescaled model (17) is a weak coupling approximation.

\section{B. Autocorrelations and space-time patterns for strong and weak chaos}

In this section we discuss the question of whether the difference between strong and weak chaos can be identified from the laser time series itself. Figure 7 shows two example trajectories (intensity traces) of a single laser with self-feedback for the regimes of weak and strong chaos. For both strong and weak chaos, there is a characteristic structure of high peaks that is significantly higher for weak chaos than for strong chaos. This may be caused by the larger coupling strength.

Figure 8 shows the laser trajectories for weak and strong chaos in space-time diagrams where the vertical axis denotes the number of the current delay window of length $\tau$ and the horizontal axis denotes the time offset $t$ in this delay
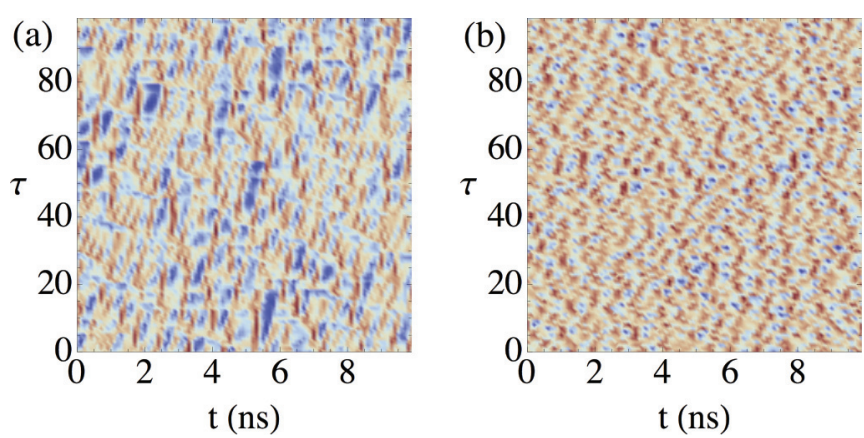

FIG. 8. (Color online) Space-time diagram of a laser trajectory (reddish, high intensity; bluish, low intensity) for a single laser with a self-feedback of $\tau=10 \mathrm{~ns}$ for (a) weak chaos $\left(\sigma=21 \mathrm{~ns}^{-1}\right)$ and (b) strong chaos $\left(\sigma=6 \mathrm{~ns}^{-1}\right)$ vs the number of the current delay window of length $\tau$ (vertical axis) and the time offset $t$ in this window (horizontal axis). 

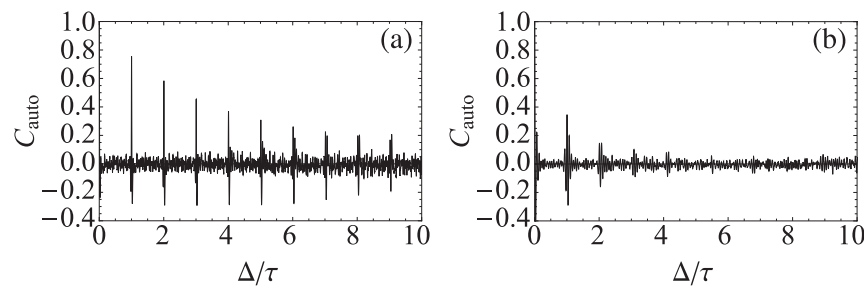

FIG. 9. Time-shifted autocorrelations $C_{\text {auto }}$ of a single laser with a self-feedback of $\tau=10 \mathrm{~ns}$ for (a) weak chaos $\left(\sigma=21 \mathrm{~ns}^{-1}\right)$ and (b) strong chaos $\left(\sigma=6 \mathrm{~ns}^{-1}\right)$ vs time shift $\Delta$ in units of the delay time $\tau$.

window. In such a representation, the exponential separation of chaotic trajectories evolves horizontally in the space direction for strong chaos. This is due to the divergence between two nearby trajectories on the internal time scale of the laser, which is much shorter than the delay time $\tau$. In contrast, weak chaos evolves vertically in the (discrete) time direction since the divergence between two nearby trajectories happens on the long time scale of the delay. Accordingly, the islands of high intensity (red) extend vertically farther in the time direction for weak chaos than they do for strong chaos. In both kinds of visualization (intensity trace and space-time diagram), however, one cannot strictly distinguish between strong and weak chaos in an unambiguous qualitative way.

Figure 9 depicts the time-shifted autocorrelations $C_{\text {auto }}$ of a single laser with self-feedback for the regimes of weak and strong chaos. For weak chaos, one clearly sees the high autocorrelation peaks at multiples of the delay time $\tau$. Although for strong chaos the chaotic behavior evolves predominantly on the internal time scale of the laser and for weak chaos it evolves on the time scale of the delay, there are non-negligible autocorrelations after multiples of the delay time $\tau$ even for strong chaos. However, they are significantly smaller than for weak chaos and decay faster with increasing time shift $\Delta$.

Considering the autocorrelations $C_{\text {auto, } \Delta=\tau}$ after one delay time $\tau$ in Fig. 10(a), we observe that they do not decay for large delay times with increasing $\tau$ but remain constant, not only for weak chaos but also for strong chaos. In Fig. 10(b) we depict the autocorrelations $C_{\text {auto, } \Delta=\tau}$ with a time shift of one delay time $\tau$ dependent on the coupling strength $\sigma$. There is no sharp transition at the critical coupling strengths.

We conclude that a linear measure such as the autocorrelation function cannot clearly uncover the difference between strong and weak chaos. Instead, we propose the usage of nonlinear measures in order to detect a more significant differ-
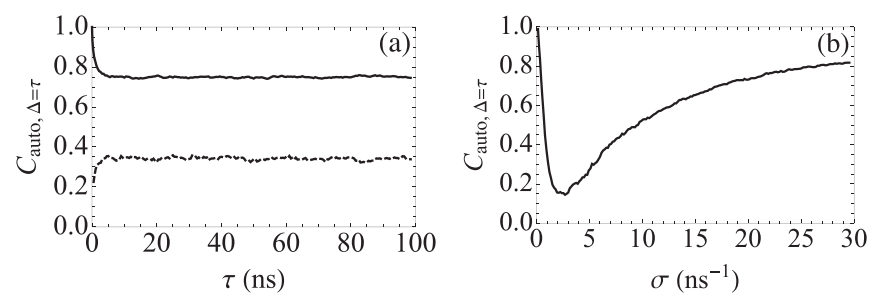

FIG. 10. (a) Autocorrelations $C_{\text {auto }}$ with a time shift of $\Delta=\tau$ for a single laser with self-feedback for weak chaos $\left(\sigma=21 \mathrm{~ns}^{-1}\right.$, solid line) and strong chaos ( $\sigma=6 \mathrm{~ns}^{-1}$, dashed line) vs delay time $\tau$. (b) Autocorrelations $C_{\text {auto }}$ with a time shift of $\Delta=\tau$ for a single laser with a self-feedback of $\tau=10 \mathrm{~ns}$ vs coupling strength $\sigma$. ence in the relationship between $\mathbf{x}(t)$ and $\mathbf{x}(t-\tau)$. The idea is motivated by the fact that in weak chaos for sufficiently large delay times, the system can be considered to be in a state of generalized synchronization with its own time-delayed feedback, whereas in strong chaos the state of the system is formally independent of its input. Note that this independence does not imply the total absence of linear correlations, as we have demonstrated. Hence the detection of strong and weak chaos from time series can be reduced to the problem of detecting generalized synchronization $[23,24]$. We refer to the relevant methods used in this context, such as the evaluation of nearest neighbors [25], mutual information, or transfer entropy [26]. However, we assume severe computational difficulties regarding memory usage and run-time connected with the necessary delay embedding. Additionally, it might even be impossible to detect generalized synchronization from a finite time series.

\section{External cavity modes for strong and weak chaos}

We can relate the strongly or weakly chaotic behavior of a laser subjected to delayed feedback to the properties of the external cavity modes (ECMs) of the laser. These ECMs are rotating wave solutions of the LK equations of the form $E(t)=$ $E_{0} e^{i \omega t}$ and $n(t)=n$ with constant amplitude $E_{0}$, frequency $\omega$, and carrier density $n$ of the laser. The spectrum of ECMs is often represented in the $(\omega, n)$ plane. In this plane the ECMs lie on an ellipse. External cavity modes located on the lower half are focus solutions called modes. The solutions located on the upper half of the ellipse are saddle points also referred to as antimodes.

Depending on the laser parameters, the ECMs have different stability properties. In the chaotic regime, the ECMs can be seen as the skeleton of the chaotic attractor $[27,28]$. In the low-frequency fluctuation regime, which occurs for low pump currents and moderate to strong coupling strengths, the intensity slowly increases, followed by a sudden power dropout. During the buildup process, the trajectory travels along the modes in the direction of the maximal gain mode, until the trajectory is expelled along the unstable manifold of an antimode. This causes the power dropout. In the coherence collapse regime, the dynamics can be described as a chaotic itinerancy between modes and antimodes.

In the long delay limit, the characteristic equation of a steady state, such as the ECM solutions, has two types of solutions, which show a different scaling behavior with the delay time [18]. The strongly unstable spectrum consists of isolated points, which are approximated by the unstable eigenvalues of the Jacobian of the LK equations without delayed terms. These eigenvalues do not scale with the delay. Besides this strongly unstable spectrum, the characteristic equation has an infinite number of solutions, forming the pseudocontinuous spectrum. The real part of these solutions scales inversely with the delay.

One can thus distinguish between strongly and weakly unstable ECMs in an analogous way as we distinguish between strong and weak chaos. The local eigenvalues of the Jacobian without delay terms play a role similar to the sub-LE $\lambda_{0}$. Strongly unstable ECMs have unstable local eigenvalues and thus a strongly unstable spectrum. The maximal eigenvalue is approximated by these local eigenvalues and does not change with the delay. The weakly unstable (and the stable) 

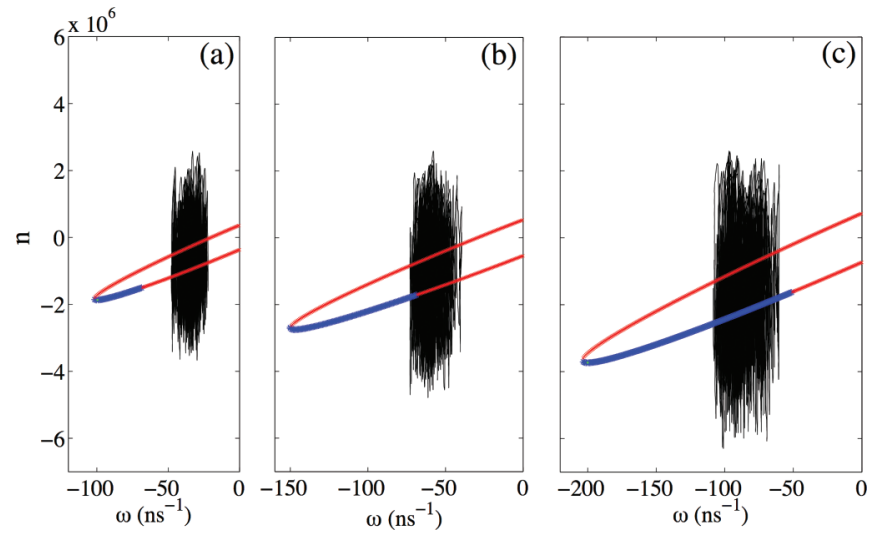

FIG. 11. (Color online) Projection of the laser dynamics onto the $(\omega, n)$ plane for a delay time $\tau=10 \mathrm{~ns}$ and a pump current $p=1.10$ Thick blue dots denote weakly unstable or stable ECMs and medium thick red dots represent strongly unstable ECMs. (a) Strongly chaotic trajectory for $\sigma=20 \mathrm{~ns}^{-1}$. The modes and antimodes involved in the dynamics are strongly unstable. (b) Dynamics around the transition point between strong and weak chaos shown for $\sigma=29.28 \mathrm{~ns}^{-1}$. The trajectory approximates the transition point between weakly and strongly unstable modes on the ellipse. (c) Weakly chaotic laser dynamics for $\sigma=40 \mathrm{~ns}^{-1}$. All the modes involved in the dynamics are weakly unstable.

ECMs have stable local eigenvalues. The strongly unstable spectrum does not exist in this case; these ECMs only have a pseudocontinuous spectrum. Hence the real part of the maximal eigenvalue scales inversely with the delay, just like the maximal LE $\lambda_{m}$ for weak chaos [29].

In Fig. 11 we show the projection of the laser dynamics onto the external cavity modes. In the strongly chaotic regime, all the modes involved in the dynamics are strongly unstable, as illustrated in Fig. 11(a). Around the transition point between strong and weak chaos, a few (two or three) weakly unstable modes are involved in the dynamics, as shown in Fig. 11(b), while most of the modes involved in the dynamics are weakly unstable in the weakly chaotic regime, as shown in Fig. 11(c). These features are independent of the pump current. The antimodes are always strongly unstable, in both the weakly and strongly chaotic regimes.

\section{Sub-Lyapunov exponents for two self-feedbacks with time-scale-separated delays}

Until now we have considered only a single laser with selffeedback and have discussed the sub-LE in this context. Now we generalize our investigation by introducing a second selffeedback with a much smaller delay. The linearized equation describing the maximal LE is

$$
\dot{\delta} \mathbf{x}=D F(\mathbf{x}) \delta \mathbf{x}+\sigma_{s} D H\left(\mathbf{x}_{\tau_{\mathbf{s}}}\right) \delta \mathbf{x}_{\tau_{\mathbf{s}}}+\sigma_{l} D H\left(\mathbf{x}_{\tau_{1}}\right) \delta \mathbf{x}_{\tau_{1}},
$$

where $\tau_{s}$ and $\tau_{l}$ are the shortest and longest feedback delays, respectively. Additionally to the sub-LE $\lambda_{0}$ [defined by Eq. (4)] we introduce another sub-LE $\lambda_{0, s}$ defined by

$$
\dot{\delta} \mathbf{x}_{\mathbf{0}, \mathbf{s}}=D F(\mathbf{x}) \delta \mathbf{x}_{\mathbf{0}, \mathbf{s}}+\sigma_{s} D H\left(\mathbf{x}_{\tau_{\mathrm{s}}}\right) \delta \mathbf{x}_{\mathbf{0}, \mathbf{s}, \tau_{\mathbf{s}}} .
$$

Thus, for $\lambda_{0, s}$ we consider a subsystem that includes only the shorter self-feedback. As before, the inserted dynamics $\mathbf{x}(t)$ is the trajectory of the full system.
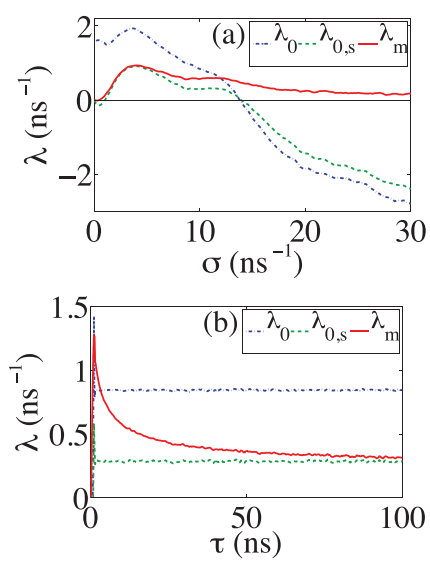

FIG. 12. (Color online) Maximal LE $\lambda_{m}$ (solid line) and sub-LEs $\lambda_{0, s}$ and $\lambda_{0}$ (dashed and dotted lines) for a single laser with two selffeedbacks $\tau_{l}$ and $\tau_{s}$ for (a) $\tau_{l}=10 \mathrm{~ns}, \tau_{s}=0.1 \mathrm{~ns}$, and $\sigma_{s}=5 \mathrm{~ns}^{-1}$ vs coupling strength $\sigma:=\sigma_{l}$ and (b) $\tau_{s}=0.1 \mathrm{~ns}, \sigma_{s}=5 \mathrm{~ns}^{-1}$, and $\sigma_{l}=10 \mathrm{~ns}^{-1}$ (strong chaos) vs delay time $\tau:=\tau_{l}$.

Figure 12(a) shows the sub-LEs $\lambda_{0}$ and $\lambda_{0, s}$ together with the maximal LE $\lambda_{m}$ for the case when $\tau_{l}$ is much larger than $\tau_{s}$. We observe that $\lambda_{0, s}<\lambda_{0}$ holds for strong chaos and $\lambda_{0, s}>\lambda_{0}$ for weak chaos. Furthermore, for large $\sigma$ both sub-LEs change their signs at approximately the same coupling strength and can hence both be used as an indicator for strong or weak chaos there. For small $\sigma$, however, only $\lambda_{0, s}$ changes its sign, indicating the transition between weak and strong chaos. Thus $\lambda_{0, s}$ is the new relevant sub-LE in a system with two timescale-separated delays. This is confirmed by Fig. 12(b), which shows that for strong chaos, $\lambda_{m} \rightarrow \lambda_{0, s}$ holds for increasing $\tau_{l}$. For weak chaos, we find $\lambda_{m} \sim 1 / \tau_{l}$ (not shown in Fig. 12).

\section{NETWORKS OF LASERS WITH TIME-DELAYED COUPLINGS}

\section{A. Master stability formalism}

We investigate complete chaotic synchronization of $N$ identical coupled laser elements. The state of each element is described by $\mathbf{x}^{i}$ with $i=1, \ldots, N$. We start from the physically motivated ansatz

$$
\dot{\mathbf{x}}^{i}=\mathbf{F}\left(\mathbf{x}^{i}\right)+\sigma \sum_{j=1}^{N} G^{i j} \mathbf{H}\left(\mathbf{x}_{\tau}^{j}\right) .
$$

The matrix $G \in \mathbb{R}^{N \times N}$ contains the network topology. Here $G^{i j}$ is the normalized coupling strength with which the laser $i$ receives input from laser $j$. In order to guarantee the existence of the completely synchronized state $\mathbf{x}^{1}(t)=\mathbf{x}^{2}(t)=\cdots=$ $\mathbf{x}^{N}(t)=: \mathbf{s}(t)$, the row sum of $G$ has to be constant for all rows and we choose $\sum_{j} G^{i j}=1$. Then the synchronized state $\mathbf{s}(t)$ reduces Eq. (20) to

$$
\dot{\mathbf{s}}=\mathbf{F}(\mathbf{s})+\sigma \mathbf{H}\left(\mathbf{s}_{\tau}\right) .
$$

The stability properties of the synchronized state $\mathbf{s}(t)$ are well described by the master stability formalism from Pecora and Carroll [30]. For completeness, we summarize the main ideas introduced in their work. A small perturbation is applied to the synchronized state such that $\mathbf{x}^{i}(t)=\mathbf{s}(t)+\delta \mathbf{x}^{i}(t)$. The 
equations of motion are then linearized around $\mathbf{s}(t)$ and the perturbations obey the equations of motion

$$
\dot{\delta} \mathbf{x}^{i}=D F(\mathbf{s}) \delta \mathbf{x}^{i}+\sigma \sum_{j=1}^{N} G^{i j} D H\left(\mathbf{s}_{\tau}\right) \delta \mathbf{x}_{\tau}^{i} .
$$

This set of equations can be decoupled into the amplitudes $\xi^{k}(t)$ of the network eigenmodes $\mathbf{g}^{k} \in \mathbb{R}^{N}$. The corresponding network eigenvalues are $\gamma_{k}$ with $G \cdot \mathbf{g}^{k}=\gamma_{k} \mathbf{g}^{k}$ and $k=$ $1, \ldots, N$. The transformation $\delta \mathbf{x}^{i}=\sum_{k} g^{k, i} \boldsymbol{\xi}^{k}$ leads to $N$ independent equations

$$
\dot{\xi}^{k}=D F(\mathbf{s}) \xi^{k}+\sigma \gamma_{k} D H\left(\mathbf{s}_{\tau}\right) \xi_{\tau}^{k} .
$$

Integration of this equation for the $k$-th perturbation mode $\mathbf{g}^{k}$ yields the maximal LE $\lambda_{k}$, which tells us about the stability of the mode, meaning that a perturbation $\delta \mathbf{x}(t)=$ $\left[\delta \mathbf{x}^{1}(t), \delta \mathbf{x}^{2}(t), \ldots, \delta \mathbf{x}^{N}(t)\right]$ in direction of $\mathbf{g}^{k}$ grows or decays exponentially. By construction, there exists at least $\gamma_{1}=1$ with the eigenvector $\mathbf{g}^{1}=(1,1, \ldots, 1)^{\top}$. It corresponds to a perturbation $\delta \mathbf{x}^{i}(t)=\delta \mathbf{s}(t), i=1 \ldots N$, within the synchronization manifold (SM). This special perturbation determines, whether the synchronous dynamics is chaotic $\left(\lambda_{1}>0\right)$ or not. The other modes correspond to linear combinations of differences between the laser elements and therefore the necessary condition to find stable complete synchronization is $\lambda_{k}<0$ for $k=2, \ldots, N$. Since all $\left|\gamma_{k}\right| \leqslant 1$, the knowledge of the master stability function $\lambda(\gamma)$ is sufficient to predict, whether a network of coupled lasers described by the coupling matrix $G$ is able to display complete synchronization or not.

\section{B. Master stability function for weak chaos}

Here we show the general master stability function for the limit of large delays in weak chaos. To this end, we make use of the scaling behavior $\lambda_{m}=O\left(\tau^{-1}\right)$. The initial point of our considerations is a generalization of Eq. (23), which reads

$$
\dot{\mathbf{y}}=A(t) \mathbf{y}+\kappa B(t) \mathbf{y}_{\tau},
$$

where $A(t):=D F[\mathbf{s}(t)]$ and $B(t):=D H[\mathbf{s}(t-\tau)]$. The exponent $\lambda_{\mathbf{y}}(\kappa)$ provides the master stability function. Transformation with $\mathbf{z}=\exp \left(-\lambda_{\mathbf{y}} t\right) \mathbf{y}$ yields a system with exponent $\lambda_{\mathbf{z}}=0$

$$
\dot{\mathbf{z}}=\left[A(t)-\lambda_{\mathbf{y}} \cdot \mathbb{1}\right] \mathbf{z}+\kappa e^{-\lambda_{\mathbf{y}} \tau} B(t) \mathbf{z}_{\tau} .
$$

Weak chaos has the important property, that by changing $A(t) \rightarrow A^{\prime}(t)=\mathbf{A}(t)+\varepsilon \cdot \mathbb{1}$ with $\varepsilon \in \mathbb{R}$ and $|\varepsilon|<\lambda_{0}$, we affect the LE only in the order of $\tau^{-1}$. Therefore, if $\varepsilon$ itself is decreasing with $\tau$, the effect on the exponent is of a smaller order in $\tau$, i.e. $O\left(\tau^{p}\right)$ with $p<-1$. Then for sufficiently large $\tau$, removing $\lambda_{\mathbf{y}}$ from the first term on the RHS of Eq. (25) leads to

$$
\dot{\mathbf{y}}^{\prime}=A(t) \mathbf{y}^{\prime}+\kappa e^{-\lambda_{\mathbf{y}} \tau} B(t) \mathbf{y}_{\tau}^{\prime},
$$

with the exponent $\lambda_{\mathbf{y}}^{\prime}=O\left(\tau^{p}\right)$. Comparison of the equivalent Eqs. (24) and (26) shows, that in the leading order of $\tau^{-1}$, rescaling of the coupling strength $\kappa \rightarrow \kappa^{\prime}=\kappa \exp \left(-\lambda_{\mathbf{y}} \tau\right)$ led to an exponent $\lambda_{\mathbf{y}}^{\prime} \approx 0$. From the knowledge of the zerocrossing at $\kappa^{\prime}$, we directly obtain the scaling law

$$
\lambda_{\mathbf{y}}(\kappa)=\frac{1}{\tau} \ln \frac{\kappa}{\kappa^{\prime}} .
$$

Although in general the value of $\kappa^{\prime}$ is unknown, this logarithmic law allows us to connect the maximal LE from within the SM with the stability of all transversal modes of a network. We refer to Eq. (23), in which $\kappa=\sigma \gamma_{k}$. Assume the maximal LE $\lambda_{m}=\lambda_{1}$ of the SM is known. It corresponds to $\gamma_{1}=1$, so $\lambda_{m}=\lambda_{1}=\lambda(\sigma 1)$ is the point at which we can fix the master stability function. Then for an arbitrary $\gamma_{k}$ we obtain from Eq. (27) the exponent

$$
\lambda\left(\sigma \gamma_{k}\right)=\lambda_{m}+\frac{1}{\tau} \ln \left|\gamma_{k}\right|
$$

Here we have made use of the fact that the exponent depends only on the absolute value $|\kappa|$ for large delays. This scaling relation also holds for steady states [14]. Transversal stability of the $k$ th mode is given if $\lambda\left(\sigma \gamma_{k}\right)<0$. This leads to the synchronization criterion

$$
\left|\gamma_{k}\right|<e^{-\lambda_{m} \tau}
$$

which connects transversal and longitudinal stability in a network exhibiting weak chaos.

\section{Master stability function for the Lang-Kobayashi dynamics}

We now investigate the master stability function $\lambda(\gamma)$ for the LK equations dynamics. For strong chaos, $\lambda(\gamma)$ is constant since the delay term becomes exponentially small in the master stability equation (23) for $\tau \rightarrow \infty$. Consequently, complete synchronization of networks with identical, strongly chaotic units is excluded on principle.

For weak chaos, $\lambda(\gamma)$ basically exhibits a logarithmic dependence on $\gamma$. However, we observe two deviations. As a first deviation, $\lambda(\gamma)$ does not diverge to $-\infty$ for $\gamma \rightarrow 0$ but has a finite value $\lambda(0)$ : the sub-LE $\lambda_{0}$. It is important to note here that the sub-LE $\lambda_{0}$ is equal to the LE of any perturbation mode with $\gamma=0$ of a network (e.g., the mode of complete synchronization of the two outer lasers in a bidirectionally coupled chain of three lasers) since then the delay term drops out in the master stability equation (23) too.

A second deviation can be considered as an effect of the finiteness of the delay time $\tau$. Figure 13 shows for several different coupling strengths and pump currents that the exponent $v$ in $\lambda \sim \ln \left(|\gamma|^{v}\right)=v \ln (|\gamma|)$ is smaller than one. We were able to find this deviation also for the Lorenz

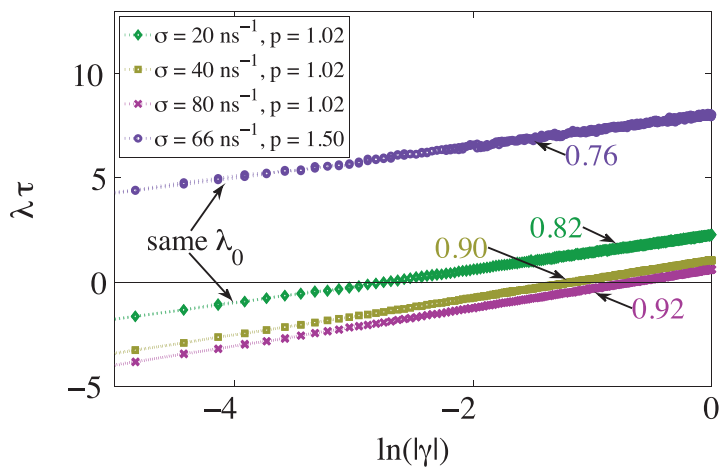

FIG. 13. (Color online) Product $\lambda \tau$ of the master stability function $\lambda(\gamma)$ and the delay time $\tau=10 \mathrm{~ns}$ for the SM and several different coupling strengths $\sigma$ and pump currents $p$ in the weak chaos regime vs $\ln (|\gamma|)$. 
dynamics. For constant delay time $\tau$ and constant laser pump current $p$, the exponent gets closer to one for larger $\sigma$, i.e., for weaker chaos. Moreover, if the coupling strength $\sigma$ is adjusted such that the sub-LE $\lambda_{0}$ is the same for two different pump currents, then the slope is closer to one for a smaller pump current. As presented in the preceding section, a condition for synchronization in a network [Eq. (29)] can be derived from the behavior of $\lambda(\gamma)$. Due to the empirically observed slope smaller than one, this condition should be refined to

$$
\left|\gamma_{2}\right|<e^{-\lambda_{m} \tau / v}
$$

where $\gamma_{2}$ is the eigenvalue of the coupling matrix $G$ with the second largest modulus.

\section{Experimental evidence of strong and weak chaos in bidirectionally delay-coupled lasers}

In a system with two bidirectionally delay-coupled lasers without self-feedback or multiple delays, identical chaos synchronization is not stable due to symmetry breaking [31]. However, chaos synchronization can still exist in the generalized sense [21]. Here we give experimental and numerical evidence that the implications of strong and weak chaos regimes also apply to the case of generalized chaos synchronization.

In the context of delay-coupled elements, correlation measures can fail to detect synchronization if the number of coupled elements is large [24]. However, the cross-correlation function is still a good indicator to identify generalized synchronization between two delay-coupled lasers. We present an example of such a cross-correlation function in Fig. 14, which shows distinct peaks at the delay time $\tau$ and its odd multiples. In this figure the large correlation peak at the delay time indicates that the lasers are generally synchronized. We argue that generalized chaos synchronization is only possible if the lasers are operating in the weak chaos regime. In contrast, a low correlation peak at a time lag $\tau$ is to be expected if the lasers are operating in the strong chaos regime [Fig. 9(b)].

Our fiber-optics-based experimental arrangement is schematically shown in Fig. 15. We use two single-mode fiber-pigtailed discrete-mode semiconductor lasers, emitting at $1542 \mathrm{~nm}$. The lasers have been hand-selected in order to achieve well-matched parameters. The laser temperatures and currents are stabilized to an accuracy of $0.01 \mathrm{~K}$ and $0.01 \mathrm{~mA}$, respectively. The lasers are biased at a current of $1.25 I_{\text {th }}$, with $I_{\text {th }}=11.7 \mathrm{~mA}$ being the solitary laser threshold current. As shown in Fig. 15, the coupling path includes two 90/10 optical

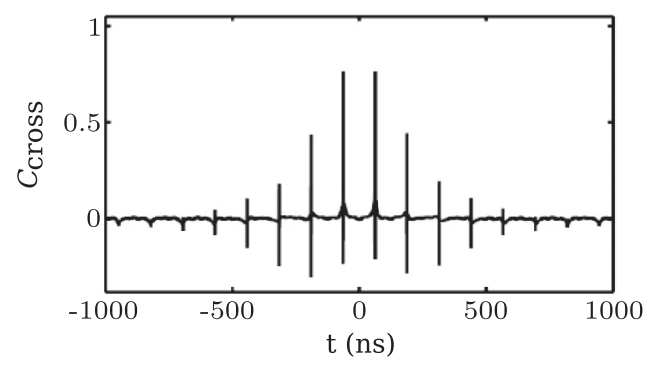

FIG. 14. Cross-correlation function of two bidirectionally delaycoupled lasers in the chaotic regime ( $\tau=63 \mathrm{~ns}$ ).

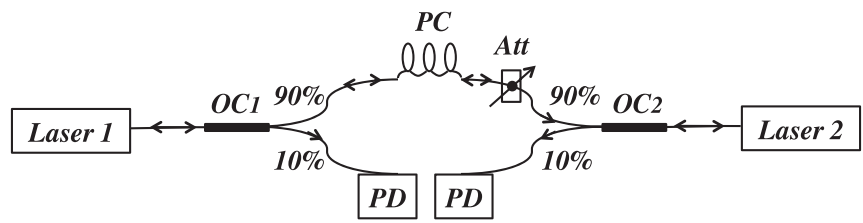

FIG. 15. Fiber-based experimental setup of two mutually delaycoupled semiconductor lasers. $\mathrm{OC}_{1,2}$ stand for the optical couplers, $\mathrm{PC}$ is the polarization controller, Att is the variable attenuator, and PD denotes the photodetectors.

couplers $\mathrm{OC}_{1,2}$, a polarization controller $\mathrm{PC}$, and an optical attenuator Att. The maximum mutual coupling obtained in this experimental arrangement can be estimated to be $\sim 40 \%$ of the emitted light. The $10 \%$ outputs of $\mathrm{OC}_{1,2}$ are used for detection.

The values of the cross correlation between the intensities emitted by lasers 1 and 2 for a time shift of $\tau$ are shown in Fig. 16(a) as a function of the coupling strength $\sigma$. For strong couplings $\sigma>0.3$, a region of large correlation is found. Decreasing the coupling strength to $0.1<\sigma<0.3$ results in a sudden decrease of the correlation. A second region of large correlation can be identified for weak couplings $\sigma \sim 0.05$. The correlation decreases again for the weakest couplings $\sigma<0.05$. The numerical results, shown in Fig. 16(b), agree with the experimental results. For large couplings, the two coupled lasers are highly correlated. A distinct region of low correlation can be seen for intermediate couplings, while the correlation increases again for the weakest coupling strengths. The numerical simulations do not reproduce the correlation decrease towards zero coupling since spontaneous emission noise sources are not considered. The numerical results of the time-shifted cross correlations for the two bidirectionally coupled lasers shown in Fig. 16(b) are similar to the autocorrelations of a single laser with self-feedback shown in Fig. 10(b). The parameters used in the numerical simulations are listed in Table II in Appendix B.

Several dynamical states are observed for variation of the coupling along the correlation curve in Fig. 16(a). The lasers operate in continuous waves with noisy fluctuations in the absence of coupling. For an increasing coupling strength, the lasers follow a quasiperiodic route via undamped
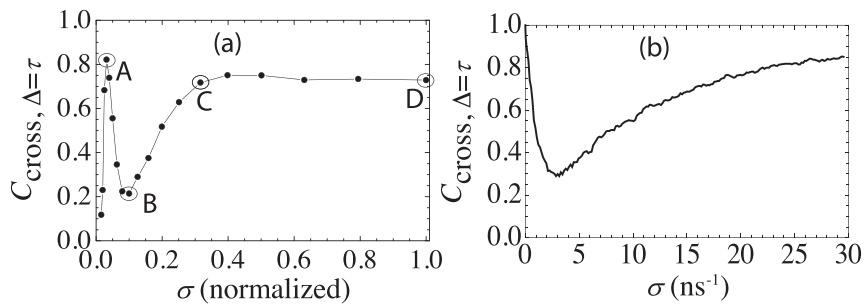

FIG. 16. (a) Experimentally measured and (b) numerically calculated cross correlations $C_{\text {cross }}$ with a time shift of $\Delta=\tau$ for a bidirectionally coupled pair of lasers as a function of the coupling strength $\sigma$. The delay time is $\tau=10 \mathrm{~ns}$ in the numerics and $\tau=63 \mathrm{~ns}$ in the experiments, corresponding to the long delay limit. The experimental coupling strength is normalized to the maximum coupling obtained in the setup, which is about $40 \%$ of the emitted light. 

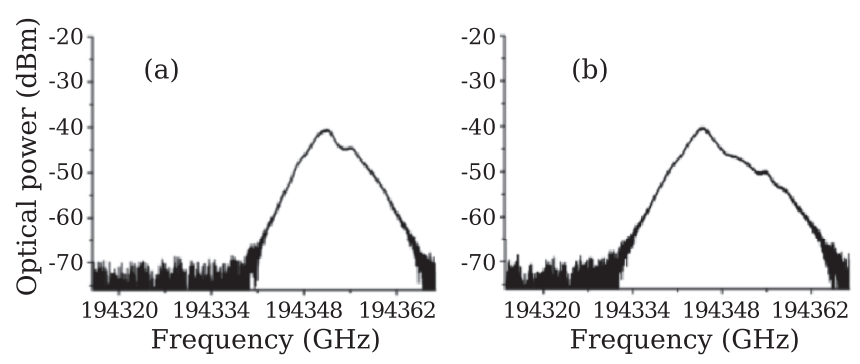

FIG. 17. Optical spectra for points denoted as (a) $B$ and (b) $C$ in Fig. 16(a).

relaxation oscillations reaching a chaotic state at point $A$. The delay-induced dynamics produces a dramatic increase in the laser optical linewidth, which increases from a few $\mathrm{MHz}$ to several $\mathrm{GHz}$ due to the coupling. We present in Fig. 17 a typical shape of the optical spectra of the chaotic laser for points denoted as $B$ and $C$ in Fig. 16(a). These two spectra are qualitatively similar apart from their different width, but correspond to significantly different correlation and synchronization properties. In order to characterize the optical spectra, we have measured their width, defined as the $-20 \mathrm{~dB}$ frequency width. The $-20 \mathrm{~dB}$ width of the optical spectrum is 9, 20, 26, and $35 \mathrm{GHz}$ for points $A, B, C$, and $D$, respectively.

We observe irregular (chaotic) dynamics in the whole range from $A$ to $D$. However, the correlation plot indicates qualitative transitions of the synchronization behavior between $A$ and $B$ and between $B$ and $C$, respectively. More precisely, the experimental correlation plot shows a large correlation around $A$ and from $C$ to $D$, indicating that the lasers are generally synchronized in these regions. The degree of synchronizability stems from the dynamical regime the lasers are operating in. Therefore, we can infer that the lasers operate in a weak chaos regime in the two high-correlation (synchronization) regions, i.e., for $\sigma>0.3$ and $\sigma \sim 0.05$. In contrast, a region of low correlation measure can be seen for intermediate couplings $\sigma \sim 0.1$. This can be associated with a strong chaos dynamical regime. The numerical correlation plot shows a similar clear distinction between different correlation regions, with a window of low correlation around $\sigma \sim 2.5 \mathrm{~ns}^{-1}$. This low correlation can be linked to a strong chaos dynamical regime.

Our experimental results on two delay-coupled semiconductor lasers presented here support the sequence of weak to strong to weak chaos with an increasing coupling strength. Even though the master stability function cannot be directly applied to the generally synchronized solution, the influence of the dynamical regime on the synchronization is clearly substantiated by our experimental and numerical results.

\section{E. Networks with several distinct sub-Lyapunov exponents}

In a network we can define a sub-LE for each individual unit. If the network is not completely symmetric then these subLEs may differ from each other, even if the units are identical. For example, in a chain of three bidirectionally coupled lasers, the middle laser is in a different coupling situation from the outer lasers. The occurrence of strong or weak chaos now depends on both sub-LEs present in the network. Figure 18(a) shows that this dependence is simple: The maximal LE $\lambda_{m}$
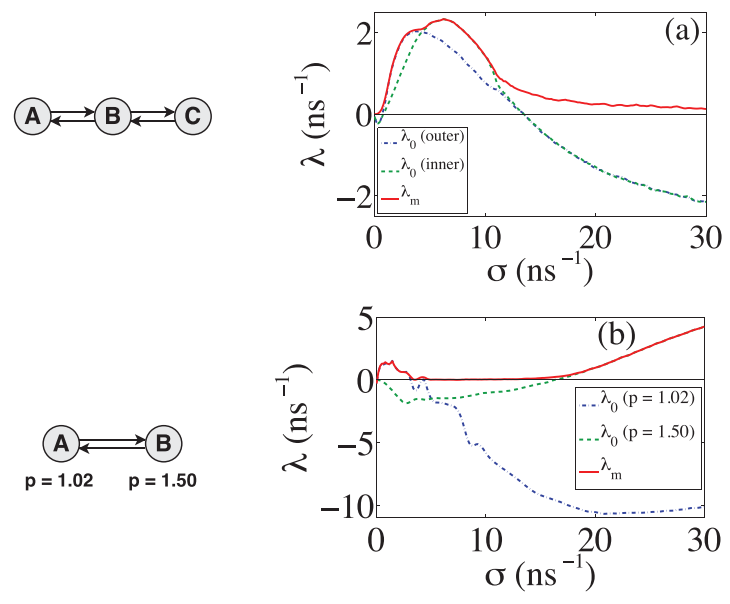

FIG. 18. (Color online) (a) Maximal LE $\lambda_{m}$ (solid line) and subLEs $\lambda_{0}$ of the inner laser (dashed line) and the outer lasers (dot-dashed line) of a chain of three lasers (see left) for a coupling delay time of $\tau=10 \mathrm{~ns}$ vs coupling strength $\sigma$. (b) Maximal LE $\lambda_{m}$ (solid line) and sub-LEs $\lambda_{0}$ (dashed and dot-dashed lines) of pair of two lasers with distinct pump currents (see left) for a coupling delay time of $\tau=10$ ns vs coupling strength $\sigma$.

of the network converges to the maximal sub-LE. This means that additional strongly chaotic units do not further increase the value of the complete network's maximal LE for strong chaos.

The individual units in the network may be nonidentical, e.g., two bidirectionally coupled lasers with different pump currents [Fig. 18(b)] $\left(p_{1}=1.02\right.$ and $\left.p_{2}=1.50\right)$. Also in this case in the strongly chaotic regime the largest sub-LE determines the maximal LE of the network. For nonidentical lasers we observe multiple transitions between strong and weak chaos for increasing coupling strength $\sigma$. These additional transitions are interrupted by periodic intervals.

As a consequence, we conclude that for large coupling delays, complete synchronization is excluded on principle for arbitrary networks that contain at least one strongly chaotic unit. Cluster synchronization between the weakly chaotic units, however, is still possible. These clusters are then driven by the strongly chaotic units.

\section{F. Sub-Lyapunov exponents for certain network patterns}

In the preceding section we have shown that identical lasers with a different number of inputs may have distinct sub-LEs. In this section we address the question of whether the number of inputs is the only criterion that determines the sub-LE for otherwise constant system parameters. We emphasize that the accumulated coupling strength of the inputs is constant for all considered network patterns.

Figure 19(a) shows a comparison of the sub-LEs of several networks where each laser receives input from exactly one laser. A single laser with self-feedback [a single-delay system (SDS)] and the bidirectionally coupled pair, for which the experimental evidence of strong and weak chaos has been provided in Sec. IV D, can be seen as limit cases of unidirectional rings containing one and two lasers. They are compared with a unidirectional ring of three lasers. Although the pair and the triangle cannot synchronize identically [Fig. 19(c)], their sub-LEs are exactly the same as the one of the SDS. 

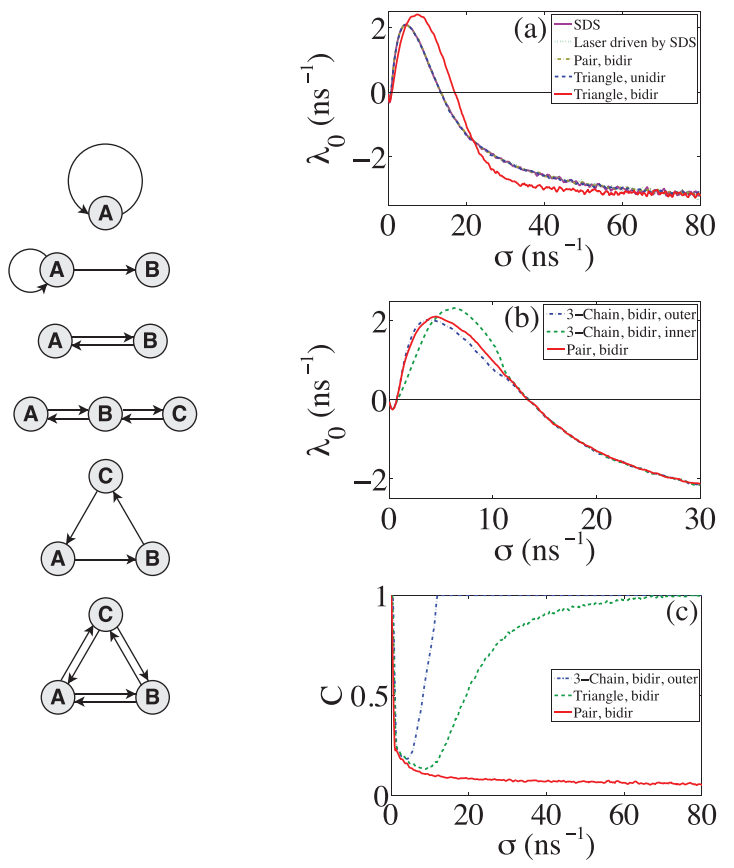

FIG. 19. (Color online) (a) Sub-LEs $\lambda_{0}$ for several network patterns where the lasers receive input from one laser (see left) in comparison with $\lambda_{0}$ of a bidirectional triangle of lasers for a coupling delay time $\tau=10 \mathrm{~ns}$ vs coupling strength $\sigma$. (b) Sub-LEs $\lambda_{0}$ of the inner and outer lasers of a chain of three lasers in comparison with $\lambda_{0}$ of a pair of lasers (see left) for a coupling delay time $\tau=10 \mathrm{~ns}$ vs coupling strength $\sigma$. (c) Cross correlation $C$ between the lasers in several network patterns for a coupling delay time $\tau=10 \mathrm{~ns}$ vs coupling strength $\sigma$.

Also the sub-LE of a laser driven by a SDS is equal to the sub-LE of the SDS, even in the strong chaos regime, when it does not synchronize to its drive. In all cases the lasers receive coherent input from a single source that exhibits the specific statistics of a laser trajectory. It does not matter if the statistics comes from a synchronized laser or an unsynchronized one, as long as it is typical for a single laser. This can also be nicely seen in Fig. 19(a) by the sub-LE of a bidirectionally coupled triangle. If the coupling strength $\sigma$ is not large enough to induce synchronization, as seen in Fig. 19(c), each laser receives incoherently superimposed input. Hence the sub-LE of the bidirectional triangle is then different from the sub-LEs of the networks with input from one laser. As soon as the bidirectional triangle synchronizes, however, its sub-LE becomes equal to the networks with input from one laser.

Figure 19(b) shows two distinct sub-LEs of the inner and outer lasers of a chain of three bidirectionally coupled lasers in comparison with the sub-LE of the bidirectional pair. If the coupling strength $\sigma$ is large enough for the outer lasers to synchronize, as seen in Fig. 19(c), then the inner laser receives the coherent superposition of the signals of the outer lasers. In consequence, the chain can be reduced to a bidirectional pair of unsynchronized lasers. Indeed, Fig. 19(b) confirms that the sub-LEs of the outer and inner lasers of the chain become identical and also equal to the sub-LE of the bidirectional pair in this regime.

Figure 20(a) shows a comparison of the sub-LEs of several network configurations (depicted on the left side) in which the
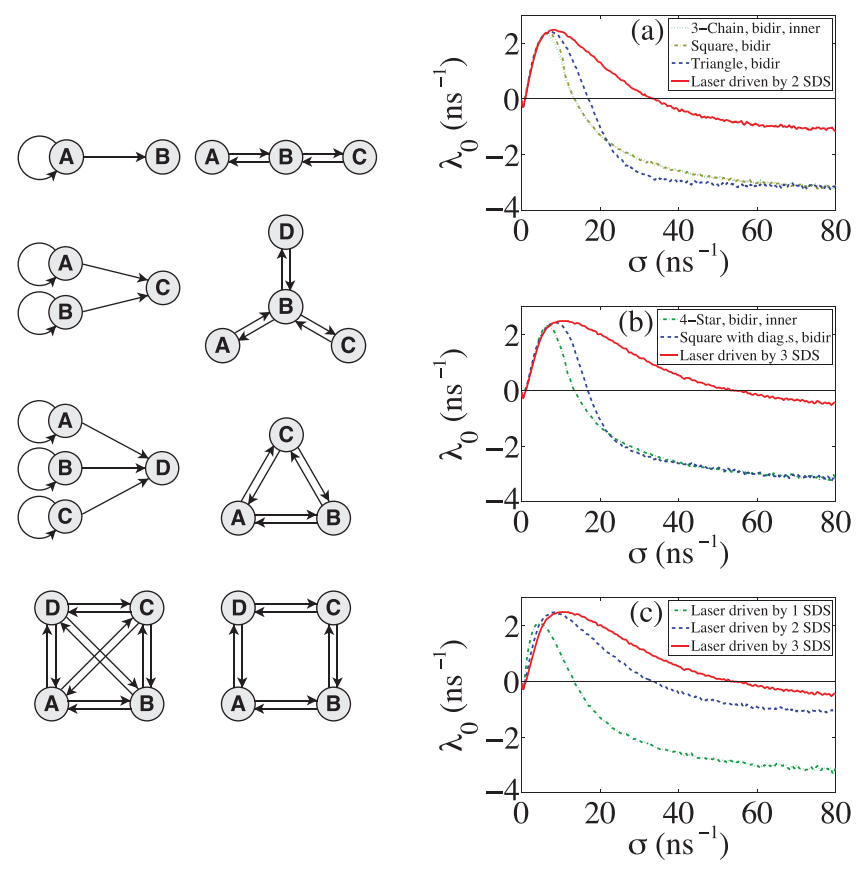

FIG. 20. (Color online) (a) Sub-LEs $\lambda_{0}$ for several network patterns where the lasers receive input from two lasers (see left) for a coupling delay time $\tau=10 \mathrm{~ns}$ vs coupling strength $\sigma$. (b) Sub-LEs $\lambda_{0}$ for several network patterns where the lasers receive input from three lasers (see left) for a coupling delay time $\tau=10 \mathrm{~ns}$ vs coupling strength $\sigma$. (c) Sub-LEs $\lambda_{0}$ of a laser driven by one, two, or three independent SDSs for a coupling delay time $\tau=10 \mathrm{~ns}$ vs coupling strength $\sigma$.

lasers receive input from two other lasers. Figure 20(b) shows a comparison of the sub-LEs of several networks (depicted on the left side) where the lasers receive input from three other lasers. In both diagrams it turns out that for small coupling strengths in a partial range of the strong chaos regime, the sub-LE indeed depends solely on the number of inputs and is equal for networks that have the same number of inputs. For larger coupling strengths, however, the sub-LEs differ from each other, as the coherence level induced by the coupling depends on the topology.

Figure 20(c) shows a comparison of the sub-LEs of a laser that receives unidirectional input from one, two, or three mutually uncoupled lasers with self-feedback. Hence Fig. 20(c) shows the dependence of the sub-LE on the number of completely incoherently superimposed inputs. For an increasing number of inputs, the properties of the received summed signal become less similar to those of a laser and increasingly similar to those of noise. We observe that with an increasing number of inputs, the sub-LE gets larger for large coupling strengths $\sigma$ and smaller for small $\sigma$.

\section{CONCLUSION}

In this paper we extended the investigations on strong and weak chaos of Ref. [17] by focusing on the dynamics of semiconductor lasers. Strong and weak chaos show different scaling properties of the maximal LE with the delay time. The sign of the artificial sub-LE $\lambda_{0}$ distinguishes between strong and weak chaos. The transition sequence of weak to strong 
chaos and back to weak chaos upon monotonically increasing the coupling strength $\sigma$ of a single laser's self-feedback was shown for numerical calculations of the LK equations. At the transition between strong and weak chaos, the sub-LE vanishes $\lambda_{0}=0$. At this transition we found $\lambda_{m} \tau \sim \sqrt{\tau}$. Transitions between strong and weak chaos by changing $\sigma$ could also be found for the Rössler and Lorenz dynamics.

Importantly, the difference between strong and weak chaos is not directly visible from the trajectory, although the difference of the trajectories induces the transitions between the two types of chaos. In addition, a linear measure such as the autocorrelation function cannot unambiguously reveal the difference between strong and weak chaos either. Although the autocorrelations after one delay time are significantly higher for weak chaos than for strong chaos, it was not possible to detect a qualitative difference. However, we could relate the trajectories of strong and weak chaos to the properties of the external cavity modes of the laser. If two time-scale-separated self-feedbacks are present, the shorter feedback has to be taken into account for the definition of a new sub-LE $\lambda_{0, s}$, which in this case determines the occurrence of strong or weak chaos. We showed that the sub-LE scales with the square root of the effective pump current $\sqrt{p-1}$, as well in its magnitude as in the position of the critical coupling strengths.

For networks of delay-coupled lasers, we explained using the master stability formalism the condition $\left|\gamma_{k}\right|<e^{-\lambda_{m} \tau}$ for stable chaos synchronization. Hence synchronization of any network depends only on the properties of a single laser and the eigenvalue gap of the coupling matrix. The master stability function for the LK dynamics was refined for a better prediction of synchronization. We provided experimental evidence of strong and weak chaos in bidirectionally delay-coupled lasers, which supports the sequence of weak to strong to weak chaos. For networks with several distinct sub-LEs it was shown that the maximal sub-LE of the network determines whether the network's maximal LE scales strongly or weakly with increasing delay time. As a consequence, complete synchronization of a network is excluded for arbitrary networks that contain at least one strongly chaotic laser. Finally, we showed that the sub-LE of a driven laser depends on the number of incoherently superimposed inputs from desynchronized input lasers.

\section{ACKNOWLEDGMENTS}

We thank the Deutsche Forschungsgemeinschaft and the Leibniz-Rechenzentrum in Garching, Germany, for their support of this work. M.C.S., I.F., and T.J. acknowledge support from MICINN (Spain) and FEDER (EU) under Projects DeCoDicA (No. TEC2009-14101) and FISICOS (No. FIS2007-60327).

\section{APPENDIX A: PARAMETERS FOR THE SIMULATION OF THE LANG-KOBAYASHI EQUATIONS}

Unless stated otherwise in the text, the constants listed in Table I were used in the simulation of the LK equations.

TABLE I. Constants used in the simulation of the LK equations. Values are taken from Ref. [32].

\begin{tabular}{lcl}
\hline \hline Parameter & Symbol & \multicolumn{1}{c}{ Value } \\
\hline linewidth enhancement factor & $\alpha$ & 5 \\
differential optical gain & $G_{N}$ & $2.142 \times 10^{4} \mathrm{~s}^{-1}$ \\
laser frequency & $\omega_{0}$ & $2 \pi c /(635 \mathrm{~nm})$ \\
pump current relative to $J_{\text {th }}$ & $p$ & 1.02 \\
threshold pump current & & \\
$\quad$ of solitary laser & $J_{\text {th }}$ & $\gamma N_{\text {sol }}$ \\
carrier decay rate & $\gamma$ & $0.909 \times 10^{9} \mathrm{~s}^{-1}$ \\
carrier number of solitary laser & $N_{\text {sol }}$ & $1.707 \times 10^{8}$ \\
cavity decay rate & $\Gamma$ & $0.357 \times 10^{12} \mathrm{~s}^{-1}$ \\
\hline \hline
\end{tabular}

\section{APPENDIX B: CRITICAL COUPLING STRENGTHS DEPENDENT ON THE PUMP CURRENT}

Table II lists the critical coupling strengths for which $\lambda_{0}=0$ and at which the transitions from weak to strong chaos and from strong to weak chaos appear, dependent on the pump current $p$.

TABLE II. Critical coupling strengths where $\lambda_{0}=0$ and at which the transitions from weak to strong chaos $\left(\sigma_{\text {crit, } 1}\right)$ and from strong to weak chaos $\left(\sigma_{\text {crit }, 2}\right)$ happen, dependent on the pump current $p$.

\begin{tabular}{ccc}
\hline \hline$p$ & $\sigma_{\text {crit }, 1}\left(\mathrm{~ns}^{-1}\right)$ & $\sigma_{\text {crit }, 2}\left(\mathrm{~ns}^{-1}\right)$ \\
\hline 1.02 & 0.80 & 13.44 \\
1.05 & 0.96 & 20.80 \\
1.10 & 1.44 & 29.28 \\
1.15 & 1.76 & 35.68 \\
1.20 & 2.08 & 40.64 \\
1.25 & 2.40 & 45.12 \\
1.30 & 2.72 & 48.96 \\
1.35 & 2.88 & 52.64 \\
1.40 & 3.20 & 56.16 \\
1.45 & 3.36 & 58.88 \\
1.50 & 3.68 & 61.92 \\
\hline \hline
\end{tabular}

[1] R. Albert and A.-L. Barabási, Rev. Mod. Phys. 74, 47 (2002).

[2] S. Boccaletti et al., Phys. Rep. 424, 175 (2006).

[3] A. Arenas et al., Phys. Rep. 469, 93 (2008).

[4] C. Song et al., Science 327, 1018 (2010).

[5] R. Vardi et al., Europhys. Lett. 97, 66002 (2012); I. Kanter et al., ibid. 93, 66001 (2011).
[6] M. Lakshmanan and D. V. Senthilkumar, Dynamics of Nonlinear Time-Delay Systems (Springer, Berlin, 2010).

[7] W. Just et al., Philos. Trans. R. Soc. London Ser. A 368, 303 (2010).

[8] J. D. Farmer, Physica D 4, 366 (1982).

[9] S. Lepri et al., Physica D 70, 235 (1994).

[10] G. Giacomelli and A. Politi, Phys. Rev. Lett. 76, 2686 (1996). 
[11] T. Erneux, Applied Delay Differential Equations (Springer, New York, 2009).

[12] E. Klein, N. Gross, M. Rosenbluh, W. Kinzel, L. Khaykovich, and I. Kanter, Phys. Rev. E 73, 066214 (2006).

[13] I. Fischer, R. Vicente, J. M. Buldu, M. Peil, C. R. Mirasso, M. C. Torrent, and J. Garcia-Ojalvo, Phys. Rev. Lett. 97, 123902 (2006).

[14] V. Flunkert, S. Yanchuk, T. Dahms, and E. Scholl, Phys. Rev. Lett. 105, 254101 (2010).

[15] A. Argyris et al., Nature (London) 438, 343 (2005).

[16] I. Kanter, E. Kopelowitz, and W. Kinzel, Phys. Rev. Lett. 101, 084102 (2008)

[17] S. Heiligenthal, T. Dahms, S. Yanchuk, T. Jüngling, V. Flunkert, I. Kanter, E. Scholl, and W. Kinzel, Phys. Rev. Lett. 107, 234102 (2011).

[18] M. Lichtner, M. Wolfrum, and S. Yanchuk, SIAM J. Math. Anal. 43, 788 (2011).

[19] S. Yanchuk and P. Perlikowski, Phys. Rev. E 79, 046221 (2009).
[20] L. M. Pecora and T. L. Carroll, Phys. Rev. Lett. 64, 821 (1990).

[21] H. D. I. Abarbanel, N. F. Rulkov, and M. M. Sushchik, Phys. Rev. E 53, 4528 (1996).

[22] T. Jüngling and W. Kinzel, arXiv: 1210.3528.

[23] U. Parlitz, NOLTA IEICE 3, 113 (2012).

[24] M. C. Soriano, G. Van der Sande, I. Fischer, and C. R. Mirasso, Phys. Rev. Lett. 108, 134101 (2012).

[25] A. A. Koronovskii, O. I. Moskalenko, and A. E. Hramov, Phys. Rev. E 84, 037201 (2011).

[26] T. Schreiber, Phys. Rev. Lett. 85, 461 (2000).

[27] T. Sano, Phys. Rev. A 50, 2719 (1994).

[28] J. Mulet and C. R. Mirasso, Phys. Rev. E 59, 5400 (1999).

[29] S. Yanchuk and M. Wolfrum, SIAM J. Appl. Dyn. Syst. 9, 519 (2010).

[30] L. M. Pecora and T. L. Carroll, Phys. Rev. Lett. 80, 2109 (1998).

[31] T. Heil, I. Fischer, W. Elsässer, J. Mulet, and C. R. Mirasso, Phys. Rev. Lett. 86, 795 (2001).

[32] V. Ahlers, U. Parlitz, and W. Lauterborn, Phys. Rev. E 58, 7208 (1998). 